\title{
INFLUENCE OF PIEZOELECTRIC TRANSDUCER TO GLASS FIBER REINFORCED COMPOSITE STIFFNESS
}

\author{
Witold Rządkowski', Karol Suprynowicz' ${ }^{1}$, Paweł Pyrzanowski ${ }^{1}$ \\ 1 Faculty of Power and Aeronautical Engineering, Institute of Aeronautics and Applied Mechanics, Warsaw \\ University of Technology, Nowowiejska 24, 00-665 Warsaw, Poland, e-mail: wrzadkowski@meil.pw.edu.pl; \\ ksuprynowicz@meil.pw.edu.pl; pyrzan@meil.pw.edu.pl
}

Received: 2015.07.15

Accepted: 2015.08.05

Published: 2015.09.01

\begin{abstract}
The main goal was to determine if transducers based on piezoelectric materials are suitable for strain calculations in thin GFRP specimens. Numerous experimental studies, both physical and numerical, performed by the authors, have shown that there is a huge influence of bonded piezoelectric transducer on the overall stiffness of the measured object. The paper presents tensile test performed on strength machine with Digital Image Correlation strain and deflection observations. Test were compared with FEM models for detailed investigation. The main conclusion is piezoelectric transducers has huge influence on local stiffness of measured object. That is critical especially when they are used as strain sensors, when presence of sensor is influencing to measured results.
\end{abstract}

Keywords: piezo material, stiffness, piezoelectric strain sensor.

\section{INTRODUCTION}

In recent years piezoelectric materials are increasing their share in multiple fields of engineering applications. Their main advantage in sensor mode, when due to external excitation electric charge is generated, is independence from external power sources. Very high stiffness of piezoelectric ceramic materials gives in result very small deformations when load is applied. This results in a high resonance frequency, which, generally, is very desired in dynamic applications. Thereby they are very suitable for high frequency and impact load registration. On the other hand, it is hard to use them in small frequency load observations, due to electrical drift and energy dissipation by amplifiers.

The most important property due to reverse inverse piezoelectric effect is possibility to use them as actuators, where in combined sensor-actuator mode they can work as intelligent dumping [1] or adaptive wing geometry systems [2]

The most common used piezoelectric material PZT besides many advantages as high mechanical-electrical coupling factor, high permittivity and piezoelectric charge coefficient, they have also disadvantages like high fragility. They can be embedded inside composite structure to monitor the stress/strain concentration levels at the critical locations [3] or to delamination detection [4]. On the other hand, it is hard to use them in small frequency load observations, due to electrical drift and energy dissipation by amplifiers [5]. In that case also high stiffness may be undesired property, especially when thick and no so rigid structures are monitored. In this paper investigation of piezo transducer for local strain distribution and global stiffness of GFRP probes is presented.

\section{RESEARCH OBJECTIVE}

Research objective was to determine the influence of piezo sensor on stiffness of GFRP probes. Strain measurements obtained on piezo transducer also have to be investigated, when locally strain values are influenced by that sensor.

Four different types of GFRP probes were prepared for tests. All of them were made from 


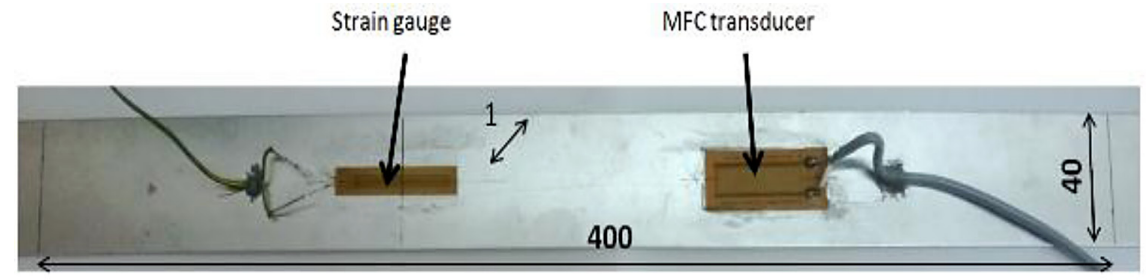

Fig. 1. Tested specimen

3 layers of glass fabric. Reinforcement consist of $186 \mathrm{~g} / \mathrm{m}^{2}$ glass fabric and epoxy EP52 was used as a matrix.

Also a probe from aluminum alloy was prepared as a reference, due isotropic material properties. On all the probes MFC (Macro Fiber Composite) M2807-P1 $(28 \times 7 \times 0.1 \mathrm{~mm})$ and resistive strain gauge with active area $30 \times 3.8 \mathrm{~mm}$ were bonded. Simple tension test were performed on Instron strength machine and all results were registered with National Instruments data acquisition system. Nikon digital camera with open source software prepared by Karol Suprynowicz [6]. On Figure 1 reference, aluminum alloy specimen with bonded transducers, before DIC markers application is presented.

Schematic represantation of fabric orirntation is shown on Figure 2. Overall stiffness of specimen is the highes for PPP samples, and lowest for

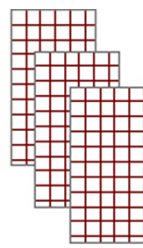

$$
\text { PPP }
$$

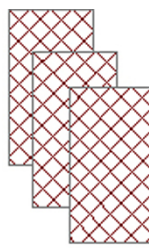

SSS

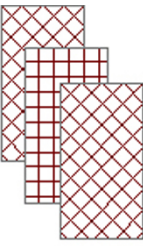

SPS

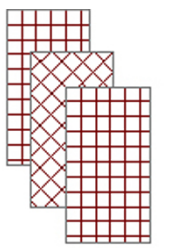

PSP
Fig. 2. Fabric orientation: $\mathrm{P}$ - fabric layer with $0 / 90^{\circ}$ orientation, $\mathrm{S}-$ fabric layer with $-45 / 45^{\circ}$ orientation

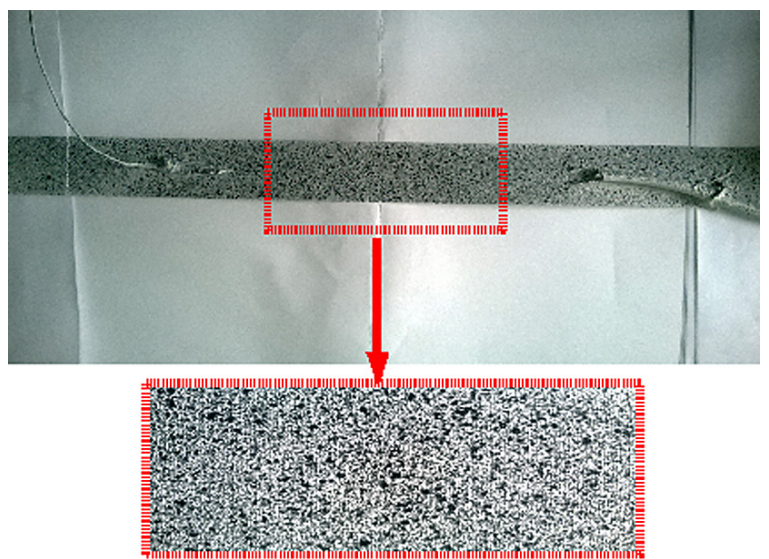

Fig. 3. DIC markers on specimen described as SSS. When both piezo and resistive gauge transducers are bonded DIC markers were applied. Sub-pixel, two dimensional DIC [7] was used to determine stain distribution and for attaching virtual extensometrs

\section{TESTS AND RESULTS}

Simple tension test were performed, where applied deflection was $0.85 \mathrm{~mm}$. Strain calculated from the formula:

$$
\varepsilon=\frac{\Delta l}{l_{0}}
$$

is equal to $0.2125 \%$, when $1_{0}$ is $400 \mathrm{~mm}$ and is very similar as calculated on DIC extensometer, when extensometer is applied above piezo sensor. It is worth to see that deflection at the end point and all intermediate points are consistent. Deflections at end point are presented on Figure 4.

Deflection along Y axis is very close to theoretical in every region of specimen and no significant disruption is observed. Locally there is a strain concentration observed on the area of MFC transducer. Vertical $(\mathrm{Y})$ strain results for specimen PPP are presened on Figure 5.

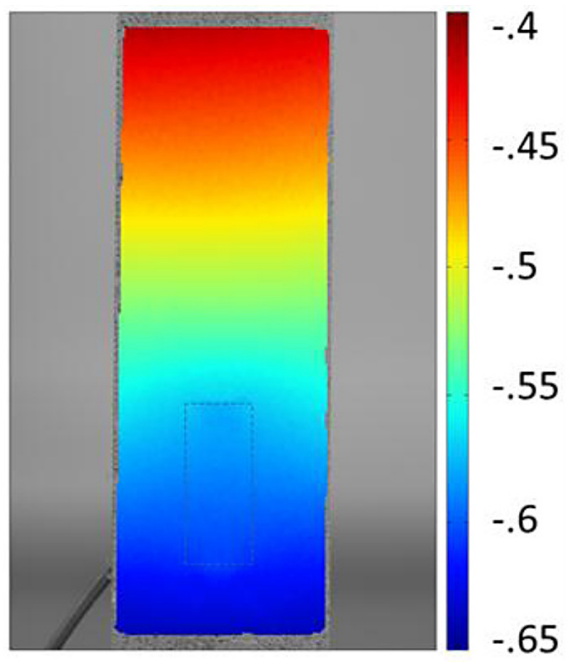

Fig. 4. Vertical (Y) deflection in PPP specimen 


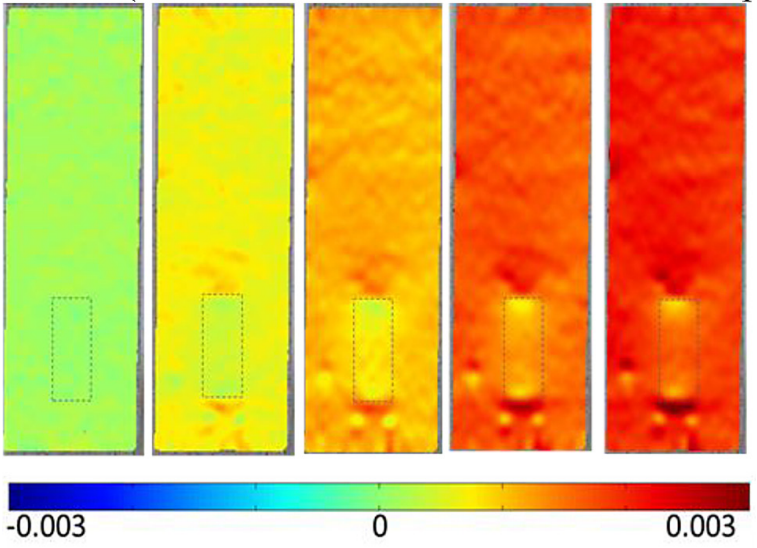

Fig. 5. Vertical (Y) strain in PPP specimen

The calculated strain values in the area of piezo sensor are significantly lower than those calculated from the analytical formulas and calculated by DIC in the area above the sensor. Two points below MFC transducer, where strain is also lower there are soldering points that should not be taken into consideration.

When displacements are also taken into consideration it leads to a conclusion that stiffness is locally higher, if deflections are continuous and strains are lower.

Numeric test were also performed for detailed investigation. FEM model has not the same geometric properties as the model used in the test, because FEM model was prepared to test new design of piezo actuator [8], but the influence of piezo material for local and global stiffness is also easy to show. According to greater piezo thickness in FEM model $(0.8 \mathrm{~mm}$ in FEM vs. $0.3 \mathrm{~mm}$ in experiment) that influence should be even more easily noticeable. In Figure 6 the results of strain i vertical direction (y) are presented. On the left the results for the side with bonded piezo transducer are shown and on the right of the opposite side. It is easy to observe that local strain distribution is highly disrupted in the surrounded area of the piezo transducer. On second side this influence is still visible, but it is not so huge as on the front side.

PPP probes are the stiffest when they are loaded in Y direction, and stiffness drops when $S$ layers number increased. The results for specimen SSS - less stiff in tension $(\mathrm{Y})$ direction are presented on Figure 7.

Strain values in the area of piezo transducer in SSS samples measured by DIC method are lower than for PPP probes. It is easier to determine if the results are combined together. In

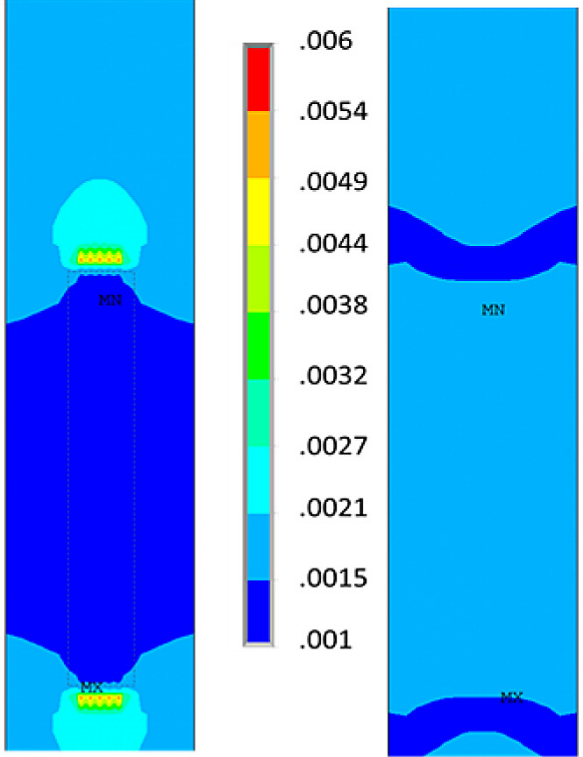

Fig. 6. Vertical (Y) strain in PPP specimen on front(left side) and back (right side)
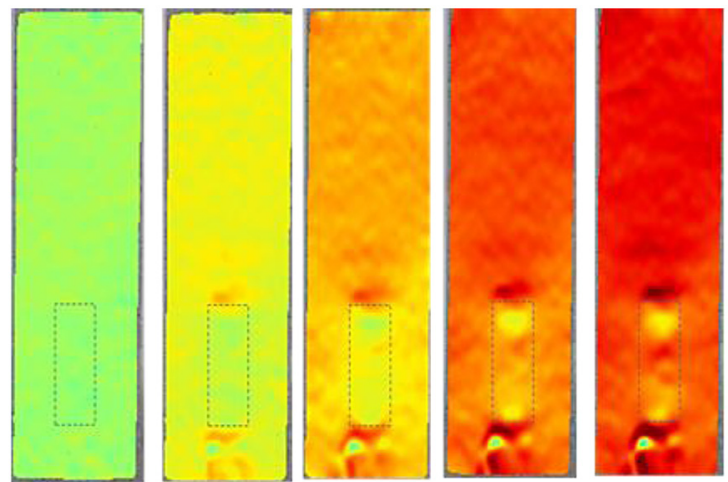

$-0.003$

0

0.003

Fig. 7. Vertical $(\mathrm{Y})$ strain in SSS specimen

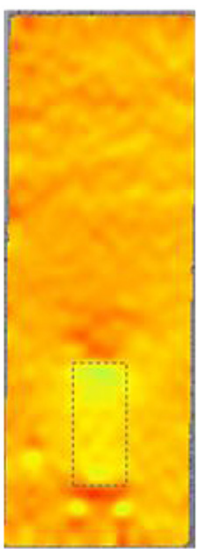

PPP
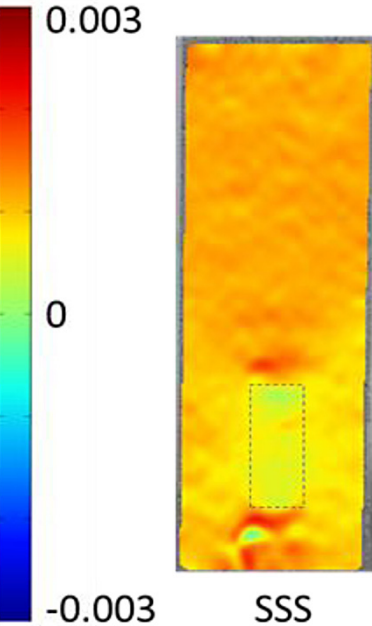

SSS
Fig. 8. Vertical (Y) strain in PPP and SSS samples 


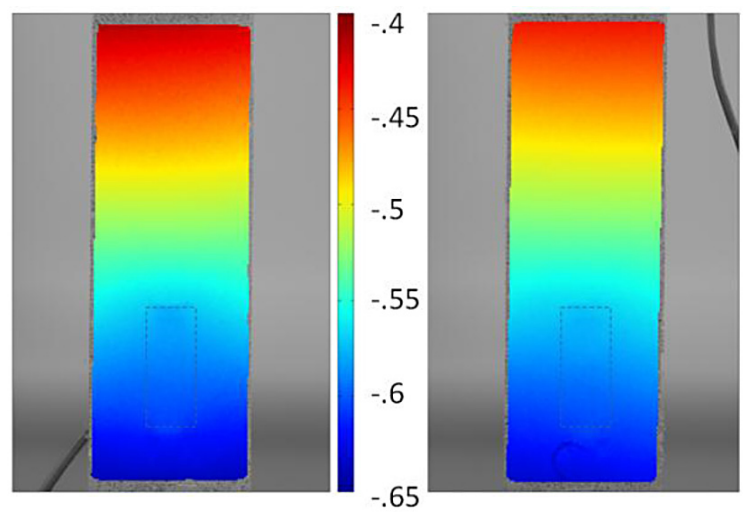

Fig. 9. Vertical (Y) deflection in PPP and SSS samples
Figure 8 the measurements from middle of load cycle are presented.

The amount of strain field in yellow and green color is higher in SSS probe, but it is necessary to take deflection distribution in SSS sample into consideration, which are shown in Figure 9.

Deflection distribution in SSS sample is the same as in PPP sample. So the only reasonable justification is local stiffness change in the area of sensor. That change has not only a result in starain maps in probes, it also influences the obtained strain values from piezo sensor. The re-
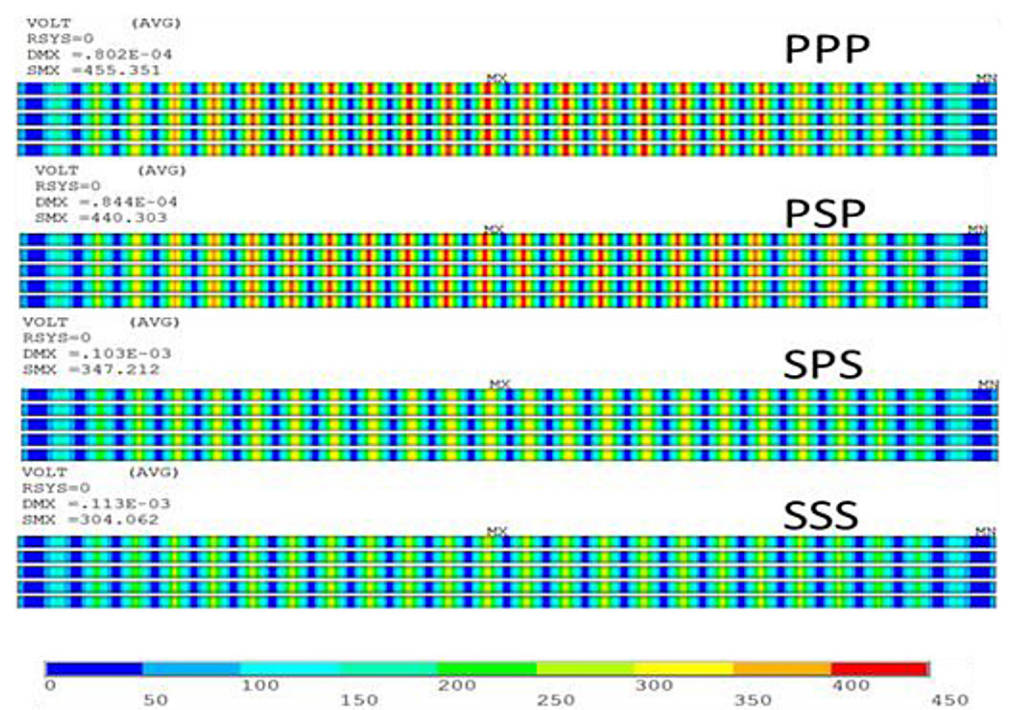

Fig. 10. Voltage values in 4 types of samples

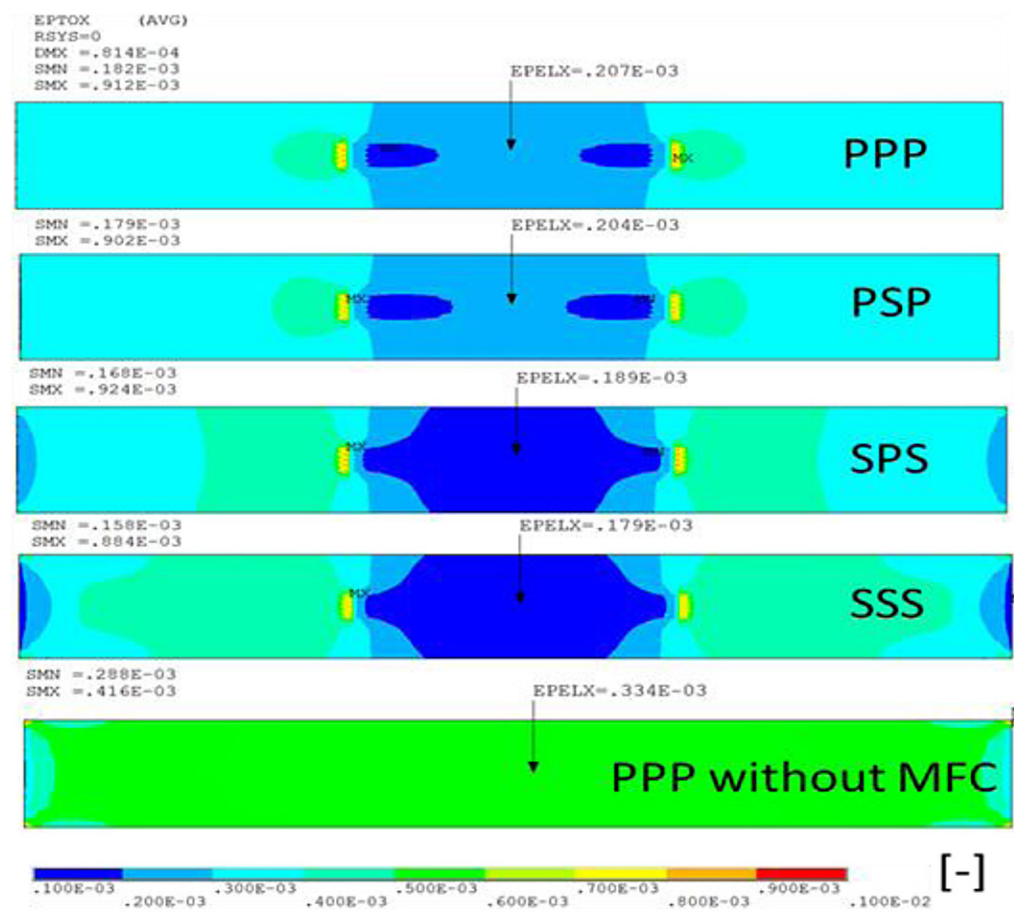

Fig. 11. Strain distribution in 4 types of samples 
sults from FEM calculations for 4 types of fabric orientation are shown on Figure 10.

Results shown clearly prove that presence of piezoelectric sensor has an influence on voltage vales obtained from piezo transducer. That voltage is dependent from strain, and presence of sensor effects that value.

It is worth to compare strain distributions in all types of specimens and investigate with probe without bonded piezoelectric transducer. In Figure 11 such results are presented. It is worth to notice that the last one (PPP without MFC) has almost constant strain distribution. Only distortion is present at ends, and is caused by boundary conditions applied in that areas. Degrees of freedom are locked at ends to simulate clamping in strength machine. In that case specimens were load by $0.05 \mathrm{~mm}$ displacement, and their overall length was 150 each. Theoretical strain calculated from $\mathrm{e}=\Delta l / l_{0}$ formula is equal to $0.33 \%$.

Both voltage values from Figure 10 and strain values from Figure 11 are summarized in the plot below. Figure 12 is a chart of both strains on the front and back of specimens with obtained voltage values (right $\mathrm{Y}$ axis). Of course, functions are not linear, due to nonlinear behavior of specimens. Bonded piezoelectric sensor changes locally location of neutral bending plane.

\section{CONCLUSIONS}

Piezoelectric materials are very interesting and useful for engineers. They can be used in many fields of modern engineering. Strain sensor is one of such use, but in that case special precautions must be taken. Piezoelectric transducers can significantly change the stiffness of the measured probes, and decrease the obtained strain values causing that the results are unreliable. This situation was observed in laboratory tests and confirmed by numerical calculations. The best solution can be made to prevent that situation - the results can be scaled by a factor to match strain values in the area not in close region to the sensor. Such an approach requires additional method to calculate real strains and in many applications it becomes unreasonable. This and other difficulties, like output signal drop in time in low frequency loads, makes sensors based on piezoelectric ceramics material are still no first choice in strain measurements.

\section{Acknowledgements}

Financial support of Structural Funds in the Operational Programme - Innovative Economy (IE OP) financed from the European Regional Development Fund - Project "Modern material technologies in aerospace industry", No POIG.01.01.0200-015/08-00 is gratefully acknowledged.

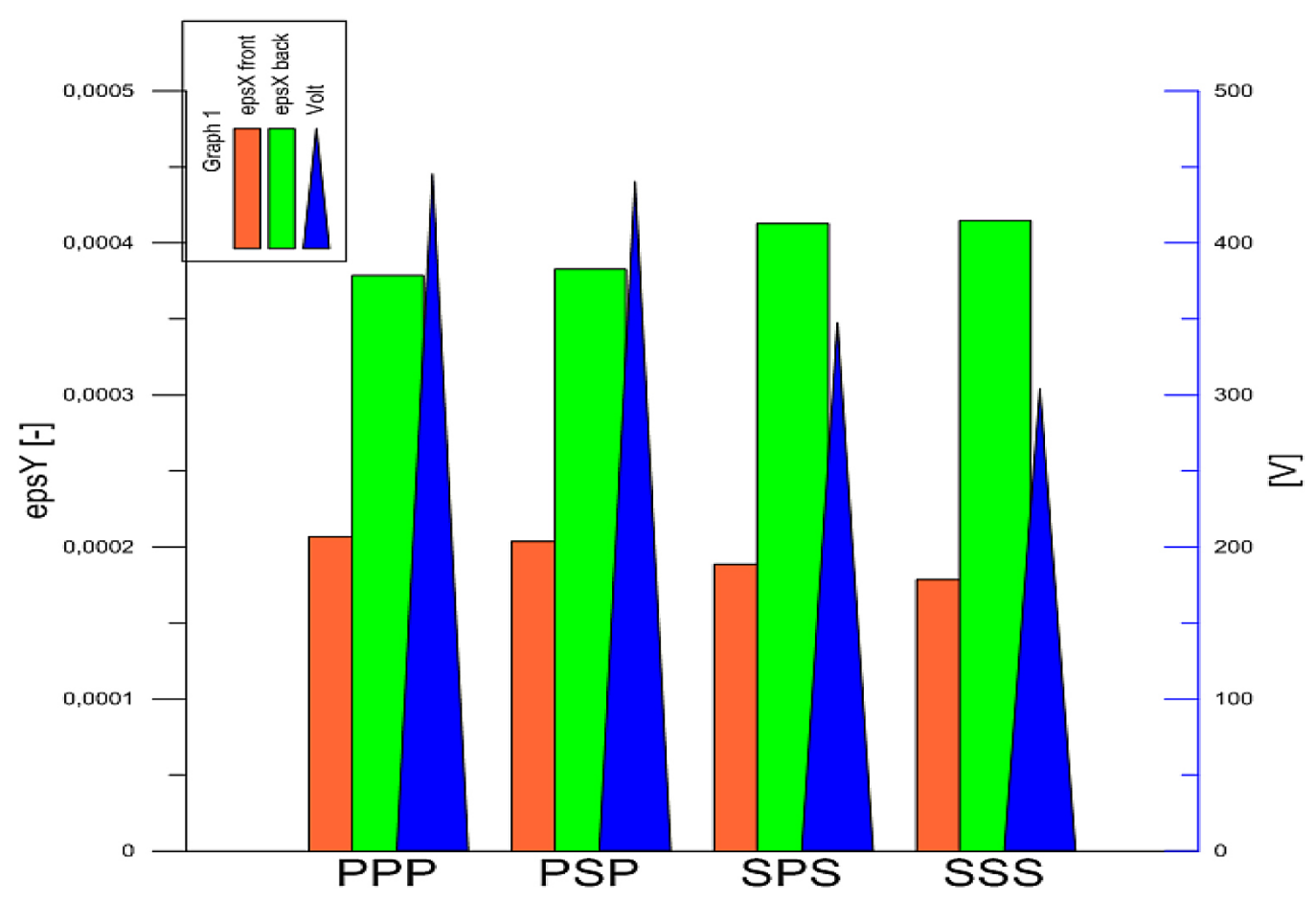

Fig. 12. Voltage and strain in 4 types of sample loaded with same displacement value $(0.05 \mathrm{~mm})$ 


\section{REFERENCES}

1. Paradies R., Ciresa P., Active wing design with integrated flight control using piezoelectric macro fiber composites. Smart Materials and Structures, 2009.

2. Debiasi M., Leong C.W., Boureme Y., Yen Y.C., Application of Macro-Fiber-Composite Materials on UAV, 2013, online March 2015, http:// www.marcodebiasi.net/MDresearch/MDpublica-tions/2013ATS-Paper-MFCwingShaping.pdf]

3. Konka H.P., Wahab M.A., Lian K., Piezoelectric fiber composite transducers for health monitoring in composite. Sensor and Actuators A, 194, 2013, 84-94.

4. Yeum C.M., Sohn H., Ihn J.B., Lim H.J., Instantaneous delamination detection in a composite plate using a dual piezoelectric transducer network. Composite Structures, 94, 2012, 3490-3499.
5. Rządkowski W., Pyrzanowski P., Determination of damage to the piezoelectric sensor by measuring the electrical capacitance. Solid State Phenomena, Warsaw 2015.

6. Suprynowicz K., Kowalik M., Obszański M., Rządkowski W., Pyrzanowski P., Estimation of piezoelectric strain sensor influence on glass fiber reinforced composite sample's stiffness using digital image correlation. In: 30th Danubia Adria Symposium on Advenced Mechanics, Croatia 2013, 105-106.

7. Bruck H.A., McNeill S.R., Sutton M.A., Peters III W.H., Digital image correlation using Newton-Rapson method for partial differential correction. Smart Structures and Intelligent Systems, 1993, 341-352.

8. Pyrzanowski P., Rządkowski W., Suprynowicz K., A method for producing a piezoelectric sensor and piezoelectric sensor. Polish Standard No. P.409809, 2015. 